\title{
Servicio Científico Roche
}

\section{VALIUM*''Roche'*}

\author{
Collective Abstract
}

VALIUM 'Roche'

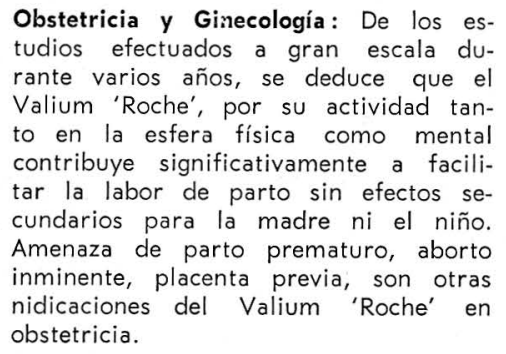

\section{VALIUM 'ROCHE' IN OBSTETRICS AND GYNECOLOGY}

\author{
EI VALIUM 'Roche' en Obstetricia y Ginecología
}

Entre las drogas utilizadas en el manejo del parto, el VALIUM 'Roche' ha llegado a desempeñar un papel cada día más importante. La relajación mental y muscular, - dos efectos deseables no sólo en obstetricia sin otambién en ginecología-, se obtienen más rápida y cabalmente que con la utilización de Librium, compuesto químicamente relacionado.

\section{Farmacología}

Al contrario de los barbitúricos, el VALIUM 'Roche', miembro de la serie de las benzodiazepinas, no actúa primordialmente sobre el neocortex, sino que ejerce su acción sobre el sistema límbico, substrato morfológico que rige el comportamiento emocional y consecuencialmente ligado à la función autónoma de los órganos. Una preparación, que como el VALIUM 'Roche', actúa sobre el sistema límbico, empleada a dosis que no produzcan efecto hignógeno, puede pues relajar y calmar a los pacientes excitados y ansiosos. Además de su efecto psicorelajante y ansiolítico, la preparación también produce una marcada relajación de la musculatura estriada mediante la inhibición de los reflejos polisinápticos.

Numerosos autores consideran que la relajación del miometrio contribuye a la regularización de las contracciones, facilitando en esta forma la labor de parto; asímismo, la acelera. Una cuantiosa evidencia sugiere que el VALIUM 'Roche' puede, en principio, acortar la duración del trabajo de parto 11, 12, 22, 25, 28, 30, 41, 89, 94. No queda duda de que el efecto calmante y relajante que ejerce, justifica su aplicación a un amplio campo en obstetricia y ginecología.

No menos importante, la ausencia de efectos secundarios graves en tratamientos prolongados y a las dosis recomendadas, confirma lo adecuado que es el VALIUM 'Roche' en el trabajo de parto.

\footnotetext{
* Marca Registrada.
} 


\section{Facilitación del parto}

Como lo testifica una abundante literatura, la gran mayoría de mujeres contempla con aprensión el momento del parto. Generalmente esto da origen a una gran variedad de reacciones psicológicas, incluyendo la tensión muscular o psíquica. (Para un más exhaustivo estudio de las cuestiones psicosomáticas implicadas, ver: Simposio de Viena, 'Músculo y psiquis' 3 y el Estudio de Hoff $y$ Ringel 40 ).

De los estudios efectuados a gran escala durante varios años, se deduce que el VALIUM 'Roche', por virtud de su actividad tanto en la esfera física como mental (efecto calmante $y$ relajante), contribuye significativamente a facilitar la labor del parto. $1,4,5,7,8,17,23,33,35,41,46$, $48,49,51,56,60,62,65,71,79$, $81,83,85,88,92,105$.

Se ha llegado a la conclusión de que el período de dilatación es el momento más apropiado para la administración. En la primípara, el efecto de la preparación (inyectada vía i.m.) llegaba a su máximo cuando el cérvix presentaba una dilatación de tres dedos. En la multípara, el VALIUM 'Roche' puede administrarse vía i.m. cuando hay dos dedos de dilatación. La dosis máxima es de 10-20 mg. (1-2 amp.) i.m. Si fuere necesario, puede aplicarse otra inyección cuando el cérvix alcance una dilatación de cinco dedos. La administración oral de comprimidos o jarabe es también efectiva, pero debe hacerse al comienzo del período de dilatación 9, 29, 42, 44, 45, $50,52,60,64,65,74,78$.

La combinación con oxitocina es posible 24, 59, 60, 81. En este caso el VALIUM 'Roche' asume una función de sustentación, proporcionando una relajación mental al administrarse antes del comienzo de las contracciones 46, 103.

La segunda etapa en ningún caso se retrasó y no se registraron anormalidades en la tercera 35, 56, 59, 72, 78, 80, 81, 83, 96. Las pacientes estaban relajadas, pero no excesivamente sedadas. Permanecieron alerta, orientadas y capacitadas para participar activamente. EI VALIUM 'Roche' no afecta el desarrollo normal del parto.

Una vez que la paciente se ve liberada del temor al dolor, seguirá las instrucciones del médico con prontitud y entendimiento. Estará aislada pero no indiferente $5,7,13$, $23,32,35,41,46,56,59,79,81$, 98,103 . La preparación no posee propiedades analgésicas, sin embargo, es posible que reduzca la percepción del dolor, de acuerdo a lo reportado por dos autores. La partureinta parece procesar el dolor con mejor voluntad bajo la influencia del VALIUM 'Roche' y así lo soporta mejor. Si la analgesia se hace necesaria, se recomienda una combinación de VALIUM 'Roche' y $1 / 2-1$ amp. de Lorfalgyl* (una ampolla de Lorfalgyl contiene $100 \mathrm{mg}$. de hidroclorhidrato de petidina y $2,25 \mathrm{mg}$. de tartrato de levallorphan). El tartrato de Levallorphan, que es un antagonista de la morfina, se utiliza para impedir la asfixia causada por los opiáceos $84,103 . * *$

La administración de VALIUM 'Roche' a las dosis prescritas no tiene efectos adversos ni en la madre ni en el niño. No hay alteraciones de

* Marca Registrada.

* * La petidina y el tartrato de levallorphan pueden administrarse mezclados. Sin embargo el VALIUM 'Roche' siempre debe aplicarse solo, ya que la solución es incompatible con la mayor parte de otras drogas. 
circulación y cualquier aumento de la presión sanguínea permanece dentro de los límites normales del parto fisiológico. Como lo señalan numerosos autores, el VALIUM 'Roche' al contrario de la mayoría de los agentes neurolépticos, no tiene un efecto hipotensivo real. Sin embargo, se ha encontrado que el alivio de la excitación y ansiedad contribuye a bajar la presión sanguínea. El ritmo cardíaco de la madre y el niño no se altera. Por último, puede agregarse, que algunos autores utilizaron el VALIUM 'Roche' sin ningún analgésico, obteniendo resultados sorprendentemente similares a los obtenidos cuando sí los utilizaron 10, 14, 23, 24, 31, 35, $41,43,53,59,60,74,79,82,84$, $98,100,103$.

\section{Amenaza de parto prematuro}

La amenaza de parto prematuro puede ser contrarrestada si la dilatación del cérvix no alcanza más de 2-3 cm. La terapia puede tener éxito siempre y cuando se establezca a tiempo. Sin embargo, y una vez hayan comenzado las contracciones éstas no podrán reprimirse $5,6,13$, 38, 41, 68, 78, 81, 82, 107.

Para el tratamiento del parto prematuro debe administrarse una dosis inicial de VALIUM 'Roche' de 10 mg. cuatro veces, o $20 \mathrm{mg}$. tres veces al día vía i.m. con dosis orales de sostenimiento durante uno o dos días. Esto produjo una gran necesidad de sueño en la paciente, efecto por demás deseable, ya que facilita la observancia de quietud total. Una vez que se haya obtenido el efecto deseado, gradualmente se reduce la dosis a cero o a un nivel de sostenimiento, según lo requieran las circunstancias. También se recomienda el uso de la preparación cuando ocurre un prematuro rompimiento de la bolsa de aguas, ya que existe la po- sibilidad de diferir el parto por unos pocos días y aún ha sido posible demorarlo por el término de semanas en algunos pocos casos 5, 6, 41, 46, 78.

\section{Aborto inminente}

En el aborto inminente el terapeuta se enfrenta con numerosos problemas. La multiplicidad de las causas, que pueden variar desde una intervención criminal hasta el caso de un embrión no viable, ilustran las dificultades involucradas en esta indicación. Pero ya que las medidas terapéuticas ofrecen posibilidad de éxito-siempre y cuando se apliquen al observar las primeras manifestaciones-, se justifica su ejecución aún antes de que se haya establecido un diagnóstico claro 68. La posología es la misma que se aplica para los casos de parto prematuro, la somnolencia y los síntomas atáxico socasionales, son efectos secundarios no perjudiciales, ya que en este caso también se necesita un reposo absoluto. Subsecuentemente, se reduce la dosis o se descontinúa el tratamiento, depende del éxito que se haya logrado. Al dar de alta a la paciente, se le debe instruir para que tome 1-2 comprimidos de VALIUM 'Roche' de 10 mg. inmediatamente sienta nuevas contracciones. Se ha confirmado unánimemente que por este medio se suprimen las contraccioens suaves en 15-20 minutos 19 . La preparación también ha sido utilizada con éxito en el aborto habitual 73,106 . En un considerable número de casos ha sido posible llevar el embarazo a su término, o por lo menos hasta asegurar la viabilidad del feto mediante el tratamiento con VALIUM 'Roche' solo o en combinación con preparaciones de progesterona o estrógeno $5,6,8,16,19,35$, $38,41,68,73,75,79,81,82,90$. 
Sin embargo, aquí, como en el caso de amenaza de parto prematuro, no es posible calcular exactamente un índice de éxitos, ya que inevitablemente las cifras incluyen casos tales como los de anomalías embrionarias, mientras que por el contrario, en cierto porcentaje de casos solo se requiere un reposo absoluto.

Cuando el proceso de Shirodkar está indicado en la insuficiencia cervical, su ejecución se hace más fácil -aun cuando la prognosis sea mala-, mediante la administración de VALIUM 'Roche' antes y después de la operación 5, 19, 20, 36, 39, 41, $54,68,79,106$. En algunos pocos casos de intervención por mioma, la expulsión del feto también fue impedida al administrar VALIUM 'Roche' y progesterona 38.

\section{Placenta previa}

En la actualidad, se opina que la placenta previa no implica necesariamente una emergencia quirúrgica. En muchos casos, el reposo es suficiente para atenuar las contracciones prematuras, y promueve la coagulación en las partes de la placenta que se hayan desprendido. Así pues, un obligado reposo en la cama frecuentemente permite que el embarazo casi llegue a su término. Se obtuvieron muy buenos resultados con VALIUM 'Roche' administrado parenteralmente a una dosis inicial de $20 \mathrm{mg}$. tres ve-

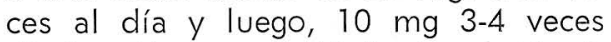
diarias (i.m. u oral) hasta el término.

\section{Ginecología}

Artner, entre otros, ha señalado los elementos psicosomáticos involucrados en las enfermedades ginecológicas de origen endocrino. Se refiere a los estudios psicofarmacológicos que demuestran la existencia de una estrecha relación entre las funciones psíquicas y autónomas centrales. Esto es particularmente cierto en los proceso psicológicos endocrinos que ocurren en la mujer sexualmente madura, "se caracterizan por una específica periodicidad o reactividad 2 . Si dicha periodicidad se ve alterada por excitaciones, ansiedades, etc., aparecerán síntomas que complican el diagnóstico si no se tiene en cuenta el estado emocional. Uno de los mejores ejemplos conocidos es el de la amenorrea temporal que se presenta en mujeres sentenciadas a prisión o en refugiadas de guerra. La naturaleza psicosomática de esta condición abre un amplio campo de indicaciones para el VALIUM 'Roche' en la práctica ginecológica. Estudios a gran escala han demostrado que la administración de la droga, logra un marcado cambio parasimpático trofotrópico ya que los sistemas del vago y del simpático están funcionalmente relacoinados. La preparación puede emplearse eficazmente cuando este último está bajo situaciones de stress de origen emocional. Entre los trastornos de esta naturaleza también se hallan el síndrome premenstrual y los disturbios autónomos de la región pelviana y de la vejiga; dismenorreas, anexopatía y espasmos de las trompas de Falopio. Los estudios efectuados hasta la fecha, han confirmado ampliamente la eficacia del VALIUM 'Roche' en estas indicaciones. Para los tratamientos ambulatorios se recomienda una dosis de 2 mg. tres veces al día, administrando dosis mayores a las pacientes con afecciones más severas y que requieran reposo absoluto. Como el VALIUM 'Roche' no tiene un efecto realmente analgésico, algunas veces puede ser ventajoso utilizarlo en combinación con un analgésico suave. 15, $17,34,53,63,91$. Una aplicación terapéutica que hasta ahora quizá no 
ha sido suficientemente aprovechada, es el tratmaeinto de los disturbios psicosomáticos durante la menopausia 21, 76.

\section{Tolerancia}

Por unanimidad, el VALIUM 'Roche' ha sido declarado como bien tolerado. La dosis requerida para el manejo de la labor normal de parto, no siempre produce una acentuada sedación en la parturienta. El efecto es frecuentemente tal, que la paciente puede dormir entre una y otra contracción pero permitiéndole participar activamente en el alumbramiento. La dosis más alta, requerida para sostener un embarazo en peligro, a menudo causa somnolencia. Sin embargo, en estos casos, este efecto concomitante es deseable ya que facilita el indispensable reposo absoluto. Pueden sobrevenir manifestaciones de ataxia y vértigo cuando se emplean dosis muy altas, pero el sistema cardiocirculatorio tanto de la madre como del niño no se ven afectados y sobre todo no se aumenta el riesgo de asfixia, aunque debe tenerse la precaución de administrar las dosis recomendadas y tener especialmente en cuenta una posible potencialización mutua, cuando se asocian otras preparaciones que actúen sobre el sistema nervioso central.

No han quedado secuelas luego de la administración de VALIUM 'Roche', aún en tratamientos de meses de duración. No ha tenido ningún efecto perjudicial sobre las funciones hepática y hemopoyética. La acción de anticoagulantes y preparaciones antidiabéticas no se afecta 11, 25, $27,29,42,55,67,74,78,81$. En el tratamiento de la dismenorrea o síndrome premenstrual, el efecto terapéutico ha sido ocasionalmente acompañado de leve somnolencia. Cuando no es necesario un descanso absoluto en la cama, este efecto concomitante no es deseable y puede contrarrestarse ajustando las dosis a las circunstancias individuales de la paciente.

\section{Resumen}

EI VALIUM 'Roche' ejerce un poderoso efecto central, dirigido principalmente al sistema límbico, centro regulador de la conducta emocional. Por virtud de su leve efecto sobre la corteza cerebral, trae consigo relajación y calma, asímismo estabilidad autónoma sin afectar el sensorio. El efecto concomitante de relajación de la musculatura estriada es muy valioso en el tratamiento de los disturbios que presentan un tono muscular elevado. (El. espasmos musculares).

La administración del VALIUM ‘Roche' durante el período de dilatación proporciona tranquilidad mental; la ansiedad intermitente $u$ otros estados de excitación más acentuados se mantienen bajo control, facilitando así el curso fisiológico del parto. Parece que es posible abreviar el período de dilatación, mientras que el período de expulsión del feto y de la placenta no se altera. Según las dosis administradas, la parturienta permanece relajada y presenta un grado mayor o menor de sedación, algunas veces con necesidad de sueño. Sin embargo, está atenta, orientada y capacitada para colaborar activamente en el alumbramiento. El recién nacido es vigoroso. No hay riesgo de asfixia empleando las dosis recomendadas. Debe tenerse en cuenta la posible potencialización si se utiliza el VALIUM 'Roche' con otras drogas de efecto central.

Administrado oportunamente, puede demorar las contracciones uterinas. Esta propiedad ha sido utilizada para sostener el embarazo en los 
casos de aborto inminente o amenaza de parto precipitado, así como en el rompimiento prematuro de la bolsa de aguas.

En c.' ecología el VALIUM 'Roche' está indicado en el tratamiento de los trastornos psicosomáticos (síndrome premenstrual, vejiga irritable, dismenorrea, adnexopatía, espasmos de las trompas, síndrome menopáusi$\mathrm{co}$ ).

\section{BIBLIOGRAFIA}

1 ARNOLD, M.: Comment on the paper: A propos du Librium au cours de I'accouchement (Stucki, D., Gross, J.). Gynaecología (Basel) 155, 122 (1963).

2 ARTNER, J.: Vegetative Querschnittsuntersuchungen nach Medikation mit Benzodiazepinderivaten; in: Relaxation und Sedation des menschlichen Uterus, pp. 77-86. Edited by W. Neuweiler, M. Berger. Basle, New York: Karger, 1964.

3 ARTNER, J.: Psyche und Ovarialfunktion; in Muskel und Psyche, pp. 215-226. Edited by $\mathrm{H}$. Hoff, $H$. Tschábitscher, K. KryspinExner, Basle, New York: Karger, 1964.

4 BAUMGARTEN, K.: Uter eine aubergwohnliche Drillingschwangerschaft. Wien. Klin. Wschr. 74, 752-753 (1962).

5 BAUMGARTEN, K.: Librium und Valium in der Schwangerschaft. Wien. Klin. Wschr. 75, 263-267 (1963).

6 BAUMGARTEN, K.: Die klinische Bedeutung der Benzodiazepine bie der Behandlung des Abortus imminene (und der drohenden Früh gerburt); in Relaxation und Sedation des menschlichen Uterus, pp. 362-374. Edited by W. Neuweiler, M. Berger. Basle, New York: Karger, 1964.

7 BEPKO, F., LOWE, E., WAXMAN, B.: Relief of the Emotional Factor in Labour with Parenterally Administered Diazepam. Obstet. and Gynec. 26, 852-857 (1965).

8 BERGER, M.: Relaxation und Sedation des menschlichen Uterus; in: Untersuchungen über die medikamentose Relaxation des menschlichen Uterus, pp. 3-50. Edited by J. Nenny, M. Berger. Basle, New York: Karger, 1963

9 BERGER, M.: Discussion; in: Relaxation und Sedation des menschlichen Uterus, pp.
92, 311. Edited by W. Neuweiler, M. Beregr. Basle, New York: Karger, 1964.

10 BERGER, M.: Die plazentare Ischamie in der Pathogenese der Toxamie; in: Relaxation und Sedation des menschlichen Uterus, pp. 118-128. Edited by W. Neuweiler, $M$. Berger. Basle, New York. Karger, M. Berger. Basle, New York. Karger, 1964.

11 BERGER, M.: Die Anwendung von 7-Chlor1,3-dihydro - 1 - methyl - 5 - phenyl-2H 1,4ibenæadiazepin-2-on (Diazepam) bei 300 Gebarenden. Arzneimittel-Forsch. 16, 1110 1113 (1966-.

12 BERGER, M.: Action du Valium en obstétrique (discussion). Bull. Féd. Soc. Gynéc. Obstét. franc. 17, 422-457 (1965).

13 BERGER, M.: Transabdominale Amniocentese. Gynaecologia (Basel) 159, 277-285 (1965).

14 BERGER, M.: Uber Früherkennung und Therapie der Spatgestose. Ther. Umsch. 23, 352-356 (1966).

15 BERGER, M., ARNOLD, M.: Therapie der uterinen Dysmenorrhoe mit "Valium". Gynaecologia (Basel) 155, 95-102 (1963).

16 BERGER, M., NEUWEILER, W.: Relaxation und Sedation des menschlichen Uterus. Geburtsh. u. Frauenheilk. 22, 1275-1277 (1962).

17 BERGER, M., NEUWEILER, W.: Die medikamentose Relaxation des menschlichen Uterus. Scientific Exhibit. 3rd World Congress of the International Federation for Gynecology. Vienna, 3-9. 9. 1961.

18 BERGER, M., NEUWEILER, W.: Zur Therapie bei Placenta praevia. Ther. Umsch. 20, 340-346 (1963).

19 BODEN, W.: Tokographische Untersuchungen über den EinfluB von "Valium" bei drohender Frühgeburt; in: Relaxation und Sedaiton des menschlichen Uterus, pp. 356-361. Edited by W. Neuweiler, M. Berger. Basle, New York: Karger, 1964.

20 BODEN, W.: Multiple Schwangerschaftspathologie -Konzeption nach Portio "Sanierung" - Carcinoma in situ und XervixverschluBinsuffiziens wahrend der Schwangerschaft - fetale Dysmaturitat. Geburtsh. U. Fravenheilk, 27, 398-401 (1967).

21 BOSCHANN. H. W.: Das Klimakterium der Frau. Symptomatik und Therapie. Z. arztl. Fortbild. 55, 69-75 (1966). 
22 BRUNNER, C.: Discussion; in: Relaxation und Sedation des menschlichen Uterus, $p$. 162. Edited by W. Neuweiler. M. Berger. Basle, New York: Karger, 1964.

23 BRUNNER, C.: Erfahrungen mit Benzodiazepin-Derivaten unter der Geburt; in: Relaxation und Sedation des menschlichen Uterus, pp. 232-238. Edited by Neuweiler, M. Berger. Basle, New York: Karger, 1964.

24 BUCHELE, F., HERRMANN, U.: Beitrag zur spasmolytischen Wirkung von "Valium" unter der Geburt. Gynaecologia (Basel) 156, 7-11 (1963).

25 CALDEYRO-BARCIA, R.: Action du Valium en obstétrique. Résumé critique. Bull. Féd. Soc. Gynéc. Obstét. franc. 17, 451-457 (1965)

26 CHALMERS, J. A.: L'emploi du Valium pour la prévention du travail prémature. Bull Féd. Soc. Gynéc. franc. 17, 476-468.

27 CHAPPAZ, G.: Action du Valium en obstétrique (discussion). Bull. Féd. Soc. Gynéc. Obstét. franc. 17, 422-457 (1965).

28 DESSARZIN, D., DUCREY, J. C., BIGLER, R., STAMM, O.: Utilisation de myorelaxants au cours de l'accouchement; in: Relaxation und Sedation des menschlichen Uterus, pp. 269-276. Edited by W. Neuweiler, M. Berger, Basle, New York: Karger, 1964.

29 DORING, G. K., KAUKA, E.: Kasuistische Mitteilung über die Gabe hoher Dosen von "Valium" Roche in der Schwangerschaft. Med. Welt (Stuttg.) 1964, 1855.

30 DOLIVO, M.: Action du Valium en obstétrique (discussion). Bull. Féd. Soc. Gynéc. Obstét. franc. 17, 422-457 (1965).

31 DUCREY, J. C.: Intéret de la tocodynamométrie externe et interne en obstétrique. Thesis, Geneva, 1962.

32 DUCREY, J. C.: VOLET, B.: Etude du Librium et de ses dérivés en obstétrique. Gynaecologia (Basel) 155, 123-124 (1963).

33 FROEWIS, J., FUCIK, F., KREMER, H. TEUBEL, R.: Vergleichende tokometrische Untersuchungen über die Wehenhemmung bei der Gebarenden mit Adrenosan, Benzodiazepin-Derivaten und Progesteron; in: Relaxation und Sedation des menschlichen Uterus, pp. 192-199. Edited by W. Neuweiler, M. Berger. Basle, New York: Karger, 1964.

34 GITSCH, E.: Zur Behandlung der Dysmenorrhoe mit Benzodiazepin-derivaten. Wien. klin. Wschr. 75, 921-924 (1963).
35 GODTS, P.: Essai d'evaluation de I'influence du Valium sur la durée de l'accouchement; in: Relaxation und Sedation des menschlichen Uterus, pp. 219-231. Edited by W. Neuveiler, M. Berger. Basle, New York: Karger, 1964.

36 GOTHE, H. D., HILFRICH, H. J.: Klinische Ergebnisse bei der Behandlung von drohenden Fehl und Frühgeburten; in: Relaxation und Sedation des menschlichen Uterus, pp. 350-355. Edited by W. Neuweiler, M. Berger. Basle, New York: Karger, 1964.

)7 HAHN, J.: Discussion; in: Relaxaiton und Sedation des menschlichen Uterus, p. 162. Edited by W. Neuweiler, M. Berger. Basle, New York; Ka:ger, 1964.

38 HAHN, J.. Vorlaufige Ergebnisse bei der Behandlung des Abortus imminens und der drohenden Frühgeburt mit Valium; in: Relaxation und Sedation des menschlichen Uterus, pp. 345-349. Edited by W. Neuweiler, M. Berger. Basle, New York: Karger, 1964.

39 HERRMANN, U.: Sedatio ndes Uterus bei Abortus imminens; in: Relaxation und Sedation des menschlichen Uterus, pp. 377383. Edited by W. Neuweiler, Basle, New York; Karger, 1964.

40 HOFF, H., RINGEL, E.: Neurovegetative Systemun d Psychosomatische Medizin. Z. Haut-u. Geschl.Kr. 20, XL I, 91-102 1966.

41 HUSSLEIN, H.: Relaxation in der Schwangerschaft und unter der Geburt; in: Relaxation und Sedation des menschlichen Uterus, pp. 166-177. Edited by W. Neuweiler, M. Berger. Basle, New York, Karger, 1964.

42 HUSSLEIN, H.: Action du Valium en obstétrique (discussion). Bull. Féd. Soc. Gynéc. Obstét. franc. 17, 422-427 (1965).

43 HUSSLEIN, H.: Emploi du Valium pendant la préparation et pendant l'accouchement. Rev. Méd. Psychosom. 4, No. especial, 9091 (1962).

44 HUTER, J.: Action du Valium en obstétrique (discussion). Bull. Féd. Soc. Gynéc. Obstét. franc. 17, 422-457 (1965).

45 HUTER, J., FISCHER BROOCKS, W.: Effets du Valium et de ses solvants sur I'amplitude, la fréquence, la durée de la contractio net sur le tonus de l'utérus de la rate gravide (energistrement isotonique, isométrique). Bull. Féd. Soc. Gynéc. Obstét. franc. 17, 463-464 (1965).

46 JANTZEN, H.: Beitrag zur Muskeirelaxation und Psychosedation subpartu. Erfabrungen mit Diazepin Preparaten. Tokographische 
Untersuchungen. Münch. med. Wschr. 105, 1397-1399 (1963).

47 JENNY, J.: Hysterotonographische Untersuchungen in der Spatschwangerschaft und unter der Geburt; in: Relaxation und Sedation des menschlichen Uterus, pp. 178191. Edited by W. Neuweiler, M. Berger, Basle, New York: Karger, 1964.

48 JENNY: Spasmolyse und Wehenschmerz. Gynaecologia (Basel) 156, 319-324 1963.

49 JUNG, H.: Zur Anwendung von Relaxation in der Geburtshilfe; in: Relaxation und Sedation des menschlichen Uterus, pp.249253. Edited by W. Neuweiler, M. Berger, Basle, New York; Karger, 1964.

50 JUNG, H.: Action du Valium en obstétrique (discussion). Bull. Féd. Soc. Gynéc. Obstét. franc. 17, 422-457 (1965).

51 KALKSCHMID, W.: Beitrag zur Behandlung der Praeklampis und Eklampsie; in: Relaxation und Sedation des menschlichen Uterus, pp. 150-154. Edited by W. Neuweiler, M. Berger, Basle, New York: Karger, 1964.

52 KASER, O.: Action du Valium en obstétrique (discussion). Bull. Féd. Soc. Gynéc. Obstét. franc. 17, 422-457 (1965).

53 KELLER, H. MASSINGER, H.: Arzneitherapie bei Schwangerschaftskomplikationen.

Münch. med. Wschr. 106. 2311-2316 (1964).

54 KEPP, R., OEHLERT, G.: Erfahrungen mit der operativen Behandlung bei wiederholten Fehl und Frühgeburten. Geburtsch. u. fravenheilk, 25, 523-530 (1965).

55 KOHLSCHMIDT, K.: 18 Monate Erfahrungen mit "Valium" Roche in der arztlichen Allgemeinpraxis. Forsch. Prax. Fortb. 17, 308310 (1966).

56 LAMBOT, P.: Recherches sur I'utilisation du Valium dans l'accouchement; ni: Relaxation und Sedation des menschlichen Uterus, pp. 260-268. Edited by W. Neuweiler, M. Berger. Basle, New York: Karger, 1964.

57 LEINZINGER, E.: Atiologie und Therapie der Spannungstoxamie; in: Relaxation und Sedation des menschlichen Uterus, pp. 102117. Edited by W. Neuweiler, M. Berger. Basle, New York, Karger, 1964.

58 LEINZINGER, E.: Relaxation bei Spannungstoxamie. Arch. Gynak, 198, 316-317 1963.

59 LEINZINGER, E., LECHNER, G., ZWINZ, G. : Geburtsverlauf bei Anwendung eines Beizo-
diazepin-Derivates; in: Relaxation und Sedation des mensschlichen Uterus, pp. 303310. Edited by W. Neuweiler, M. Berger. Basle, New York: Karger, 1964.

60 MAIER, L.: Relaxation und Geburtsverkürzung durch Benzodiazepin-derivate. Wien. klin. Wschr. 75, 611-612 (1963).

61 MALAGAMBA, G.: Paper, Verhandlungen der Deutschen Gellschaft für Gynakologie, 34. Versammlung. Hamburg, 9-13, 10. 1962.

62 MARTI, M. CORDEY, R., OBOLNSKY, W.: Die Progesteronkonzentration im Blut unter Librium und Valium bei drohender Frühgeburt oder unter der Geburt und tokographische Messungen; in: Relaxation und Sedation des menschlichen Uterus, pp. 341344. Edited by W. Neuweiler. M. Berger. Basle, New York: Karger, 1964.

63 MCALLISTER, D.: Prevalence of Common Menstrual Symptoms. Lancet 1963-11, 360.

64 MERGER, R.: Action du Valium en obstétrique (discussion). Bull. Féd. Soc. Gynéc. Obstét. franc. 17, 422-457 (1965).

65 MARGER R., MELCHOR, J. BERNARD, N.: Etude du Valium en obstétrique. Ossais cliniques. Effets sur la contraction utérine et sur la dilatation du col. Bull. Féd. Soc. Gynéc. Obstét. franc. 17, 468-472 (1965).

66 MERZ, W. R. : Action du Valium en obstétrique (discussion). Bull. Féd. Soc. Gynéc. Obstét. franc. 17, 422-457 (1965).

67 MESSMER, E.: Uber die Anwendungsmolichketten von Benzodiazepin-derivaten bei der Behandlung vegetativer. Regulationstorungen und psychomotorischer Unruhezustande. Therapiwoche 16, 1407-1410 (1966).

68 MEYBERG, J.: Zur Relaxation des Uterus wahrend der Schwangerschaft. Geburths. U. Fravenheilk, 25, 201-205 (1965).

69 MOSLER, K. H.: Discussion; in: Relaxation und Sedation des menschlichen Uterus, pp. 95-97. Edited by W. Neuweiler, M. Berger. Basle, New York: Karger. 1964.

70 MULLER, P.: Action du Valium en obstétrique (discussion). Bull. Féd. Soc. Gynéc. Obstét. franc. 17, 422-457 (1965).

71 MULLER, P., DELLENBACH, P., SWARCBERG, R.: L'action du Diazepan dans les menaces d'accouchement prémature. Bull. Féd. Soc. Gynéc. Obstét. franc. 17, 465 467 (1965). 
72 NAVRATIL, E.: Preface; in: Untersuchungen über die medikamentoses Relaxation des menschlichen Uterus, pp. 1-2. Edited by J. Jenny, M. Berger. Basle, New York: Karger, 1963.

73 NEUWEILER, W., BERGER, M.: Therapie bei vorzetigen Wehen; in: Relaxation und Sedation des menschlichen Uterus, pp. 317. 328. Edited by W. Neuweiler, M. Berger, Basle, New York: Karger, 1964.

74 NICOLAI, K. H.: Der Geburtsverlauf bei verschiedenen Methoden der Geburtsleitung eMd. Welt (Stuttg.) 1966, 377-381.

75 ORENGO, F.: Empleo de los derivados de la benzodiezepina en obstetricia. Rev. Españ. Obstet. Ginec. 1962, 464-471.

76 PEÑA, E. F.: Psychoneurotic Reactions in Gynecology and Obstetrics. West. Med. 3, 292-296 (1962).

77 PODIWINSKI, F.: Erfahrungen mit dem Benzodiazepinderivat Valium. Wien. med. Wschr. 115, 649-652 (1965).

78 POLLAK, K., CLOTTEN, R.: Erfahrungen mit einem neven Benzodiazepinpraparat bei Behandlung des vorzettigen Wehenbeginns. Med. Klin. 59, 1174-1177 (1964). den Abortus und der drohenden frühgeburt; in Relaxation und Sedation des menschlichne

79 RAUSCHER, H.: Zur Therapie des drohenUterus, pp. 336-340. Edited by W. Neuweiler, M. Berger. Basle, New York, Karger, 1964.

80 REHSTEINER, N. P.: Vergleichende Untersuchungen mit Valium und Analgetika sub partu; in: Relaxation und Sedation des menschlichen Uterus, pp. 297-303. Edited by W. Neuweiler, M. Berger. Basle, New York; Karger, 1964.

81 REIFENSTUHL, G., MAYER, H. G. K.: Die Bedeutung von Librium für die Relaxation des menschlichen Uterus. Wien. med. Wschr. 112, 608-610 (1962).

82 REIFENSTUHL, G.: Uber die Anwendung von Valium wahrend der Geburt und die Moglichketten seiner relaxaterenden Wirkung auf die Uterusmuskulatur; in: Relaxation und Sedation des menschlichen Uterus, pp. 254-259. Edited by W. Neuweiler, M. Berger. Basle, New York: Karger, 1964.

83 REVAZ, C., PAPADOPULUS, S., BOSSART, H., GETAZ, D., WEIHS, D.: Action du Valium sur la santé foetale in utero et postpartum; la microdétermination du $\mathrm{pH}$ sanguin foetal comme moyen de controle. Bull.
Féd. Soc. Gynéc. Obstét. franc. 17, 460462 (1965).

84 RIMBACH, E.: Erfahrungen mit der psychologischen Geburtsvorbereitung und Geburtsleitung an der Universitats Frauenklinik Tübingen. Fortschr. Med. 83, 933-935 (1965)

85 ROUCHY, R.: Action du Valium en obstétrique (discussion). Bull. Féd. Soc. Gynéc. Obstét. franc. 17, 422-457 (1965).

86 ROSSBOTH, W.: Uber die Wirkung von Benzodiazepin Derivaten in der Geburtshilfe in: Relaxation und Sedation des menschlichen Uterus, pp. 287-296. Edited by W. Neuweiler, M. Berger. Basle, New York: Karger, 1964.

87 SAAMELI, K.: Tokographische Untersuchungen zur Frage der medikamentosen Wehenhemmung; in: Relaxation und Sedation des menschlichen Uterus. pp. 277-286. Edited by W. Neuweiler, M. Berger. Basle, New York. Karger, 1964.

88 SALING, E.: Action du Valium en obstétrique (discussion). Bull. F d. Soc. Gyn c. Obst t. franc. 17, 422-457 (1965).

89 SAUTER, H.: Pharmakodynamische Wirkung alterer und moderner Relaxatien auf die menschliche Gebarmutter; in: Relaxation und Sedation des menschlichen Uterus, pp. 56-61. Edited by W. Neuweiler, M. Berger. Basle, New York: Karger, 1964.

90 SCHULTZE, K. W., PATT, V.: Zur Behandlung des abortus imminens mit Valium, einem Benzodiazepinpraparart. Geburtsh. u. Fravenheilk. 23, 1003-1009 (1963).

91 SEMMENCE, A. M.: Prevalence of Common Menstrual Symptoms. Lancet 1963-11, 685.

92 SPIELMANN, W.: Frage der Geburtserleichterung. Wien. Klin. Wschr. 75, 613-616 (1963).

93 STAMM, H.: Uterosedative Therapie bei drohendem Abort mit Valium; in:Relaxation und Sedation des menschlichen Uterus, pp. 375-376. Edited by W. Neuweiler, $M$. Berger. Basle, New York: Karger, 1964.

94 STAUB, H.: Zur Pharmakologie neverer uteriner Relaxatien; in: Relaxation und Sedation des menschlichen Uterus, pp. 47-55. Edited by W. Neuweiler, M. Berger. Basle, New York, Karger, 1964.

95 TAPFEER, S.: Discussion; in: Relaxation und Sedation des menschlichen Uterus, pp. 
89, 90, 94, 385. Edited by W. Neuweiver, M. Berger, Basle, New York: Karger, 1964.

96 TIMONEN, S., HAGNER, M.: Benzodiazepines in an Unselected Obstetrical Series. Ann. Chir. Gynaec. Fenn. 55, 65-68 1966.

97 TOULOUSE, R., MAFFEI, J. L.: Utilisation du Valium en obst-trique. Rev. franc. Gynéc. 60, 263-270 (1965).

98 TOULOUSE, R.: Comparrisons des dérivés de la benzodiazépine en cequi concerne la pouvoir acelérateur de l'accouchement; in: Relaxation und Sedation des meschlichen Uterus, pp. 200-218. Edited by W. Neuweiler, M. Berger. Basle, New York: Karger, 1964.

99 VOLET, B.: Actoin du Valium en obstétrique (discussion). Bull. Féd. Soc. Gynéc. Obstét. franc. 17, 422-457 (1965).

100 VOLET, B., DUCREY, J. C.: L'administration parentérale de Valium au cours de I'accouchement. Gynaecologia (Basel) 156, 288-289 (1963).

101 VOLET, B., DUCREY. J. C.: L'administration parentérale de Valium au cours de I'accouchement Ther. Umsch. 20, 484-489 (1963).
102 VOLET, B., RUFFIEUX, M.: Etude de I'action de spasmolytiques lors de provocations de l'accouchement par perfusion d'ocytociques; in: Relaxation und Sedation des menschlichen Uterus, pp. 212-218.

Edited by W. Neuweiler, M. Berger. Basle, New York, Karger, 1964.

103 WAGNER, H.: Die Bedeutung des limbischen Systems für die Geburtshilfe und Gynakologie; in: Relaxation und Sedation des menschlichen Uterus, pp. 18-29. Edited by W. Neuweiler, M. Berger. Basle, New York: Karger, 1964.

104 DE WATTEVILLE, H.: Discussion: in: Relaxation und Sedation des menschlichen Uterus, pp. 99, 312. Edited by W. Neuweiler, M. Berger, Basle, New York: Karger, 1964.

105 DE WATTEVILLE, H.: Action du Valium en obstétrique. Bull. Féd. Soc. Gynéc. Obstét. franc. 17, 445450 (1965).

106 WINKHAUS SCHINDL, J.: Habituelle Aborte bei Insuffizienz des inneren Muttermundes nach einer Portioamputation. Geburtsh u. Fravenheilk. 27, 316-318 (1967).

107 ZANDER, J.: Die Behandlung der bedrohten Schwangerschaft. Geburtsh. U. Fravenheilk. 27, 83-86 (1967). 